\title{
ANALYSIS OF FLOWER BUD DIFFERENTIATION OF JATROPHA NIGROVIENSRUGOSUS AT DIFFERENT DEVELOPMENTAL STAGES
}

\author{
XIAO, F. ${ }^{1,2}-$ WANG, X. R. ${ }^{1 *}-$ ZHAO, Y ${ }^{1,2}-\mathrm{HE}, \mathrm{H}^{1}{ }^{1}$ \\ ${ }^{1}$ Foresty College of Guizhou University, 550225 Guiyang, China \\ ${ }^{2}$ Institute for Forest Resources \& Environment of Guizhou, 550225 Guiyang, China \\ *Corresponding author \\ e-mail:wxr7211@126.com \\ (Received 27 $7^{\text {th }}$ Jun 2018; accepted $2^{\text {nd }}$ Aug 2018)
}

\begin{abstract}
The female to male flower ratio of Jatropha nigroviensrugosus, a new cultivar, is higher than that of the common J. curcas. However, the sex determination mechanism of J. curcas remains elusive. In the present study, gene expression analysis of inflorescence buds, female and male flowers, and new leaf buds of J. nigroviensrugosus was performed, using second-generation sequencing. The results demonstrated that high expression of genes related to sucrose phosphate synthase, soluble starch synthase (EC:2.4.1.21) and granule-bound starch synthase promoted the formation and development of male flowers, and the genes CUP-SHAPED COTYLEDON 2 (CUC2) and CUC3, played pivotal roles in regulating the formation and development of female flowers. The initial development of female flower primordia required cytokinins (CTK) induction, while late developmental stages only required a low concentration of CTK. The characteristic genes for floral organ development were conservatively expressed in the four tested parts. In this investigation, we not only provide genetic information regarding the differential developmental processes between female and male flowers, but also deepen our understanding of $J$. nigroviensrugosus.
\end{abstract}

Keywords: Jatropha, new cultivar, differential expressed gene, transcription factor

\section{Introduction}

Jatropha curcas L., a plant of the Jatropha genus in the Euphorbiaceae family, is an important energy-bearing tree which is extensively distributed worldwide. J. curcas has a high, untapped potential to contribute towards sustainable production of food and bioenergy, rehabilitation of degraded land, and reduction of atmospheric carbon dioxide, its potential oil productivity is $1.9 \mathrm{t} / \mathrm{ha}$, beginning the fourth year after planting (Montes et al., 2016; Dias et al., 2012). However, the low seed yield of has been a stumbling block in realizing its full potential as an ideal bioenergy crop, the small number of female flowers and a low female to male flower ratio (ranges from1:20 to 1:108) are the main factors limiting its seed yield (Gangwar et al., 2018; Ashoke et al., 2005). Breeders are mainly concerned with shortening the young growth cycle of $J$. curcas; increasing the ratio of female to male flowers; increasing oil content, seed weight, seed volume and other traits. Seed weight had positive correlation with seed length, breadth, thickness and oil content (Kaushik et al., 2007). The shortening of the breeding cycle and the increasing the number of female flowers contribute to the number of seeds, and the traits such as seed size, density and volume are important indicators affecting yield. Changing the offspring traits through interspecific hybridization in $J$. curcas, or through the method of discovery, identification, promotion and application of high-yield new varieties is a necessary way to genetic improvement. 
J. nigroviensrugosus CV Yang, a new variant of J. curcas discovered in 2005, its main features are the downward cotyledons and true leaves, bulged leaf tissues (wrinkled leaves). It has a larger number of female flowers and a higher female to male flower ratio, which means a higher seed yield, the experimental plots for many years of afforestation showed that the dry seed yield per hectare of five-year-old stands in $J$. nigroviensrugosus reached $3196.8 \mathrm{~kg} / \mathrm{ha}$, which was 6 times higher than J. curcas $(349.5 \sim 559.5 \mathrm{~kg} / \mathrm{ha})$; the oil content of the seeds in J. nigroviensrugosus was 40 42\%, which was higher than $J$. curcas $(30 \sim 40 \%$ ) (Yang et al., 2012, 2013, 2015). Comparison of the $\delta^{13} \mathrm{C}$ values and various photosynthetic indices between $J$. nigroviensrugosus and $J$. curcas, with respect to different flowering stages, demonstrated that during the leaf bud stage, J. nigroviensrugosus transported a large amount of nutrients to the leaf buds for their differentiation to flower buds. Therefore, flower differentiation is likely determined during the transformation from leaf to flower buds, and the flowering numbers may be determined during the inflorescence stage (He et al., 2016).

Flower formation has continuously been a focus in Plant developmental biology. Studies regarding sex expression and floral morphology are essential for deep understanding of breeding and for the determination of the reproductive potential of plant genotypes (De Lourdes Adriano-Anaya et al., 2016). The transcriptome describes all RNA transcribed by a certain tissue or cell at a certain developmental stage or in a specific physiological state, reflecting the overall expression pattern and regulation of genes. The female or male favorable analysis of floral development in both monoecious and dioecious plants by transcriptome profiling or microarray analysis may help to better understand flower development and the gender regulatory network (Mao et al., 2017; Gao et al., 2013; Rocheta et al., 2014). Hence, we performed high-throughput RNA sequencing (RNA-Seq) and differential analysis on the inflorescence buds, leaf buds, and male and female flowers of J. nigroviensrugosus. The present study shed light on the molecular basis of sex determination of J. nigroviensrugosus and elucidated the expression patterns of flowering-related genes prior to and following inflorescence and leaf bud differentiation, providing directions for the study of the flowering mechanism of J. nigroviensrugosus.

\section{Materials and methods}

\section{Materials}

The tested plants were collected from Qiaoma Experimental Foreast, Ceheng County, Guizhou Province, China. The place is a typical dry-hot valley climate which is situated $830 \mathrm{~m}$ above sea level, with an annual average temperature $19.2{ }^{\circ} \mathrm{C}$, rainfall $1340.7 \mathrm{~mm}$, sunshine duration $1514 \mathrm{~h}$ and annual frost-free period is 305 days. It is known as the "natural greenhouse". The soil is yellow sandy loam developed from sand shales, slightly acidic.. Three 8-year-old J. nigroviensrugosu (JCw) plants were selected for the present investigation. The following four tissues were collected from each of the three plants: inflorescence buds at the terminal vegetative shoots (JCw_p) (earlyforming adaxial bulges with a diameter $<0.3 \mathrm{~cm}$ ); slightly red new leaf buds at the branch tips (JCw_l); female flowers not yet open (JCw_f) (slightly visible white ovules showing after vertical cut); and male flowers not yet open (JCw_m) (slightly visible yellow anthers showing after vertical cut). Immediately after sample collection, wrap it 
in foil paper and put it into liquid nitrogen. Bring it back to the laboratory and transpose it in a $-80^{\circ} \mathrm{C}$ ultra-low temperature freezer.

\section{RNA extraction and transcriptome sequencing}

Total RNA was extracted from the above-mentioned tissue samples, using TRIzol (Invitrogen, USA). After passing the quality examination, the RNA samples were sent to Shanghai Meiji Biological Cooperation for sequencing from both ends with an Illumina Hiseq sequencing platform.

\section{Sequencing}

Following completion of the sequencing, the raw data were quality controlled and compared with the genome of $J$. curcas (https://www.ncbi.nlm.nih.gov/genome/915). The read counts were transformed into FPKM (expected number of fragments per kilobase of transcript sequence per million base pairs sequenced) values for gene expression analysis. The thresholds of $\mid \log _{2}$ (fold change) $\mid \geq 1$ and q-value $<0.05$ were used to determine the differential expressed genes (DEGs) in the resulting reads. The related DEGs were subjected to Gene Ontology (GO, http://www.geneontology.org/) functional enrichment and Kyoto Encyclopedia of Genes and Genomes (KEGG, http://www.genome.jp/kegg/) pathway enrichment.

\section{Results}

\section{Transcriptome sequencing and alignment}

FastQC (https://www.bioinformatics.babraham.ac.uk/projects/fastqc/) was used to perform quality control on the raw data, demonstrating a Q20 above 97\%, a Q30 above $93 \%$, and an average QC content of $43.5 \%$. HISAT2 (https://ccb.jhu.edu/software/hisat2/index.shtml) was used to align the sequenced transcriptome data of $J$. nigroviensrugosus with the genome of J. curcas (https://www.ncbi.nlm.nih.gov/genome/915), demonstrating alignment rates higher than 96\% (Table 1).

Table 1. Transcriptome sequence number of J. nigroviensrugosus and alignment rate against J. curcas genome

\begin{tabular}{c|c|c|c|c|c|c|c}
\hline Sample & Total_seq_num & Aligned_seq_num & Alignment_rate & Uniq_aligned_num & Uniq_rate & Multi_aligned_num & Multi_rate \\
\hline JCw_p1 & 61561080 & 59770212 & $97.09 \%$ & 47954394 & $77.90 \%$ & 11815818 & $19.19 \%$ \\
JCw_p2 & 68567902 & 66625809 & $97.17 \%$ & 53415546 & $77.90 \%$ & 13210263 & $19.27 \%$ \\
JCw_p3 & 59863830 & 58056039 & $96.98 \%$ & 46249509 & $77.26 \%$ & 11806530 & $19.72 \%$ \\
JCw_11 & 65790812 & 63842133 & $97.04 \%$ & 50803720 & $77.22 \%$ & 13038413 & $19.82 \%$ \\
JCw_12 & 57042156 & 55510903 & $97.32 \%$ & 44681167 & $78.33 \%$ & 10829736 & $18.99 \%$ \\
JCw_13 & 54306178 & 52727027 & $97.09 \%$ & 42234790 & $77.77 \%$ & 10492237 & $19.32 \%$ \\
JCw_f1 & 63398574 & 61833649 & $97.53 \%$ & 49485973 & $78.06 \%$ & 12347676 & $19.48 \%$ \\
JCw_f2 & 51699528 & 50312809 & $97.32 \%$ & 40301836 & $77.95 \%$ & 10010973 & $19.36 \%$ \\
JCw_f3 & 65665646 & 63948655 & $97.39 \%$ & 51294438 & $78.11 \%$ & 12654217 & $19.27 \%$ \\
JCw_m1 & 61817828 & 60212667 & $97.40 \%$ & 47821892 & $77.36 \%$ & 12390775 & $20.04 \%$ \\
JCw_m2 & 55453444 & 54036050 & $97.44 \%$ & 42977841 & $77.50 \%$ & 11058209 & $19.94 \%$ \\
JCw_m3 & 59762748 & 58184393 & $97.36 \%$ & 46342932 & $77.54 \%$ & 11841461 & $19.81 \%$ \\
\hline
\end{tabular}


Form 40.30 to 53.41 million sequences were aligned to unique positions in the genome, the alignment rates is ranging from 77.22 to $78.33 \%$, form 18.99 to $20.04 \%$ sequences is aligning to multiple positions in the genome. These indicating overall matched $J$. nigroviensrugosus sequences with the J. curcas genome. Consequently, the sequences may be analyzed as reference-based transcriptome. The small differences between the sequences indicate that the data were highly qualified to reveal differences in inflorescences prior to and subsequent to leaf bud differentiation.

\section{PCA and gene expression analyses}

The gene expression of four samples of J. nigroviensrugosus, based on the quantitative results of FPKM, was subjected to principal component analysis (PCA), using $\mathrm{R}$ (https://www.r-project.org/) which is an open source software. The results denote that the tip inflorescence buds (JCw_p) were closely related to the new leaf buds $(\mathrm{JCw} 1)$, with a PC1 value on the $\mathrm{X}$ axis of $44.4 \%$ and a PC2 value on the $\mathrm{Y}$ axis of $31.5 \%$. Three-dimensional (3D) visualization shows significantly clustered samples with no outliers, suggesting good biological repeatability (Fig. 1a). According to the DEG screening thresholds, statistical analysis of all pairs of J. nigroviensrugosus samples, there were 2995 DEGs between JCw_m and JCw_f, with 1733 upregulated and 862 downregulated genes in JCw_m; 4238 DEGs between JCw_p and JCw_f, with 2138 upregulated and 2100 downregulated genes in JCw_p; 6153 DEGs between in JCw_p and JCw_m, with 3226 upregulated and 2927 downregulated genes in JCw_p. There were 74 genes specifically expressed in JCw_f or JCw_m (Fig. 1b).

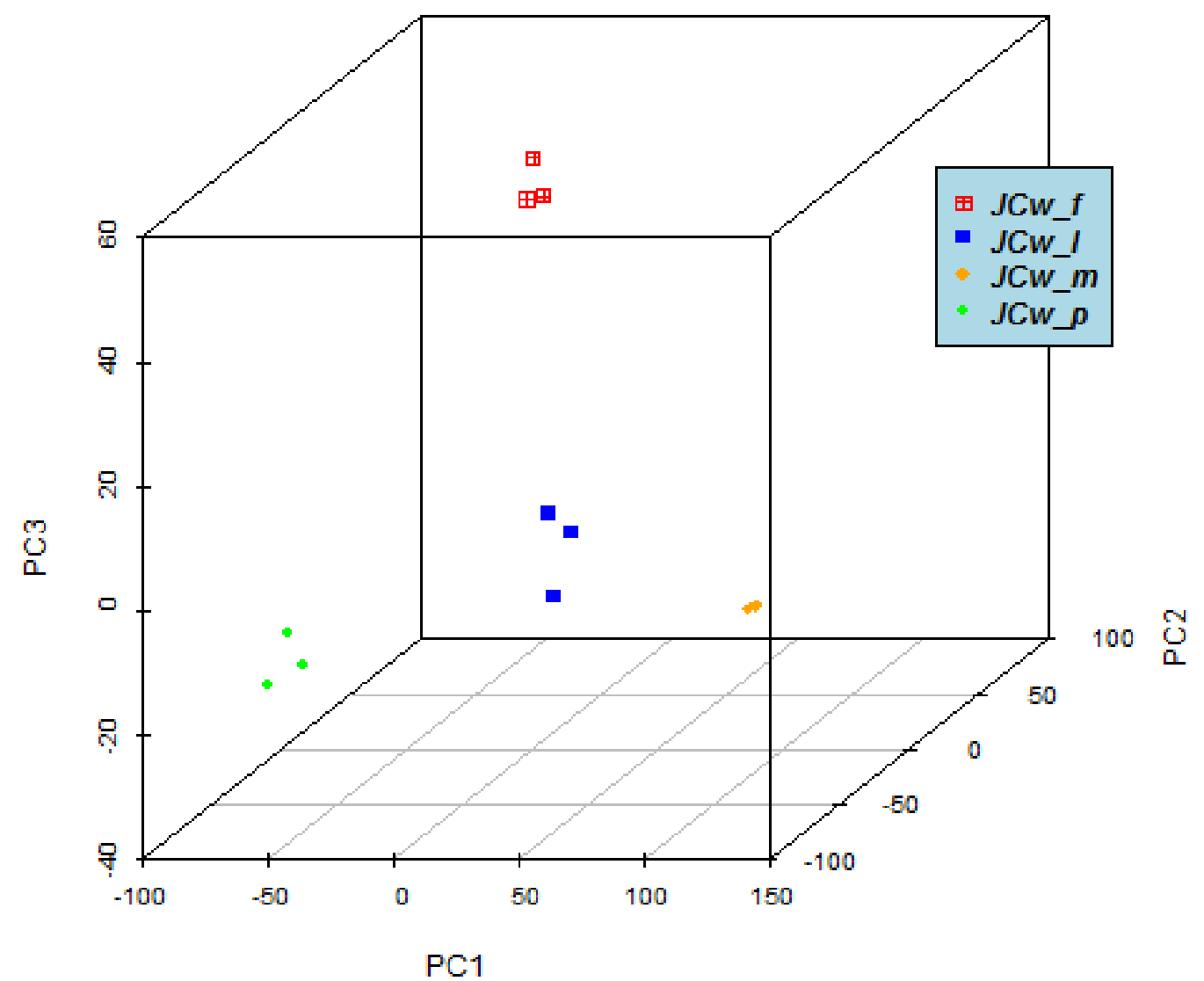




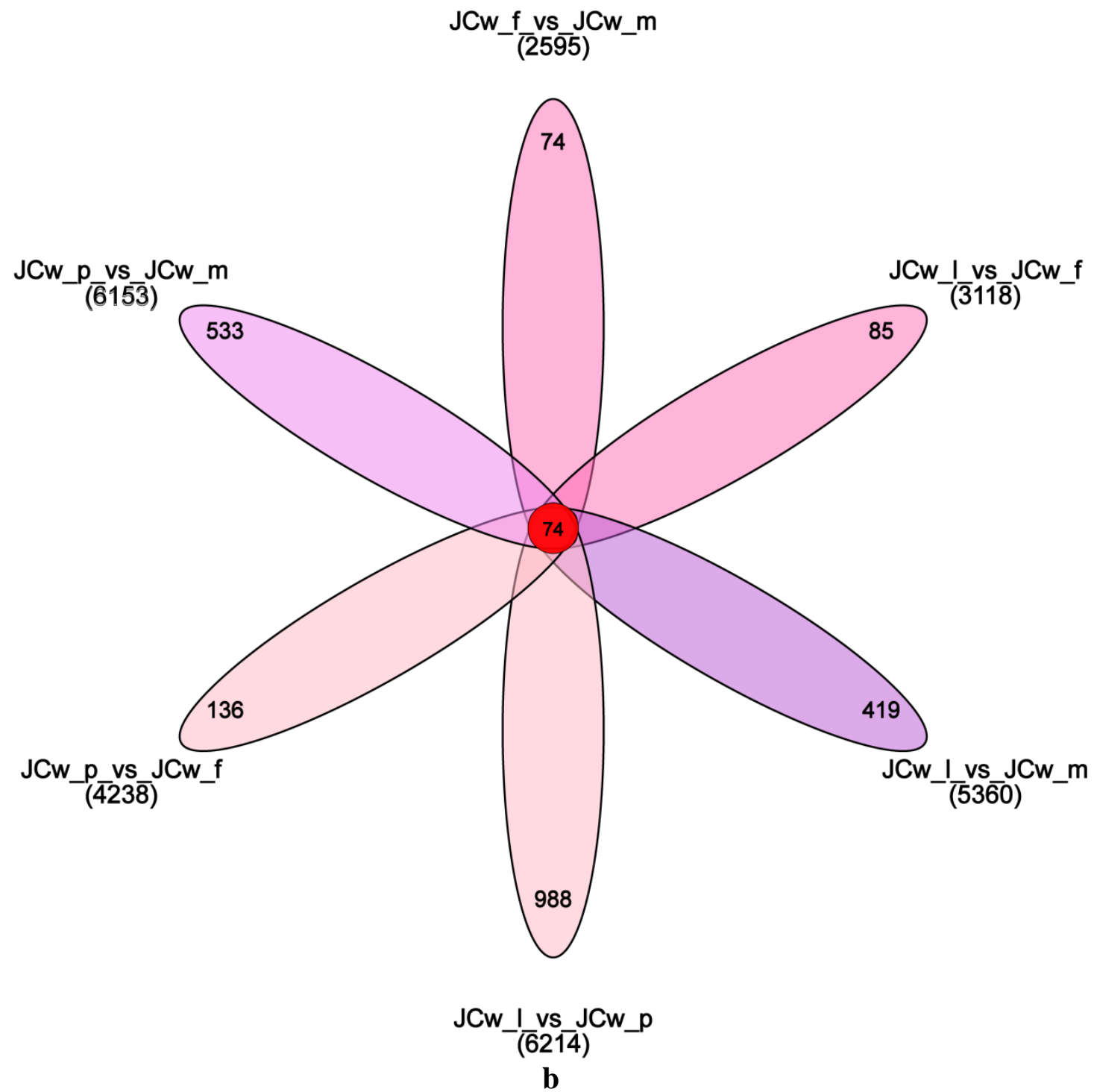

Figure 1. a. PCA of the different tissues of J. nigroviensrugosus with $3 D$ visualization. $\boldsymbol{b}$. Venn diagram of DEGs between pairs of J. nigroviensrugosus samples

\section{Functional enrichment of female and male flowers of J. nigroviensrugosus}

GO functional enrichment analysis was conducted on the DEGs between male and female flowers. With respect to the biological process, genes related to carbohydrate metabolism, including glucose, sucrose, polysaccharides and starch, were significantly enriched. With respect to the cell components, genes associated with inner and outer membranes, extracellular regions, transcription factor complexes and the cell skeleton were significantly enriched. In molecular function, genes coupled to glucosidase, hydrolase and oxidoreductase were significantly enriched. Pathway enrichment analysis of DEGs between male and female flowers (Fig. 2a) displays significantly enriched metabolic pathways such as starch and sucrose (ko00500), and pentose and glucuronic acid interconversion (ko00040). Moreover, there were significant differences in the plant hormone signal transduction pathway (ko04075), with a total of 16 hormone synthesis-related genes upregulated in female flowers and 18 upregulated in male 
flowers. The abscisic acid (ABR) synthesis-related genes SnRK2 (gene 20150) and ABF (gene 13255 and gene 14512) were significantly upregulated in male flowers. The cytokinin (CTK) synthesis-related gene A-ARR (gene 13354) was significantly upregulated in female flowers, and B-ARR (gene 20147) was significantly upregulated in male flowers.

\section{DEGs analysis between inflorescence and leaf buds, and female and male flowers}

Between inflorescence buds and male flowers, the starch and sucrose metabolic pathway (ko00500) was significantly enriched (Fig. 2b). In male flowers, the expression of 84 genes related to fructokinase (EC: 2.7.1.4) (gene 5753, gene 8905, and gene 17326), starch synthase (EC: 2.4.1.21) (gene 4801), granule-bound starch synthase (EC: 2.4.1.242) (gene 13471) and sucrose-phosphate synthase (EC: 2.4.1.14) (gene 7836, gene 12091, and gene 14471) were significantly upregulated, and 21 were downregulated. However, there was no significant enrichment of the starch and sucrose metabolic pathway between inflorescence buds and female flowers. The pathway enrichment of the DEGs between inflorescence buds and female flowers reveals enriched metabolic processes such as ribosomes, plant hormone signaling and DNA replication (Fig. 2c). With respect to the phytohormone signaling pathway, the ethylene synthesis-related gene ERF1/2 (gene 18694) was upregulated in female flowers. The pathway enrichment of the DEGs between inflorescence and leaf buds displayed enriched metabolic processes such as ribosomes, photosynthesis, photosynthesisantenna proteins, and plant hormone signaling (Fig. 2d). With respect to the photosynthetic pathway, with the exception of gamma (gene 7618) being upregulated in inflorescence buds, the remaining 48 DEGs involved in photosynthesis and 15 DEGs associated with photosynthesis-antenna proteins were all upregulated in leaf buds.

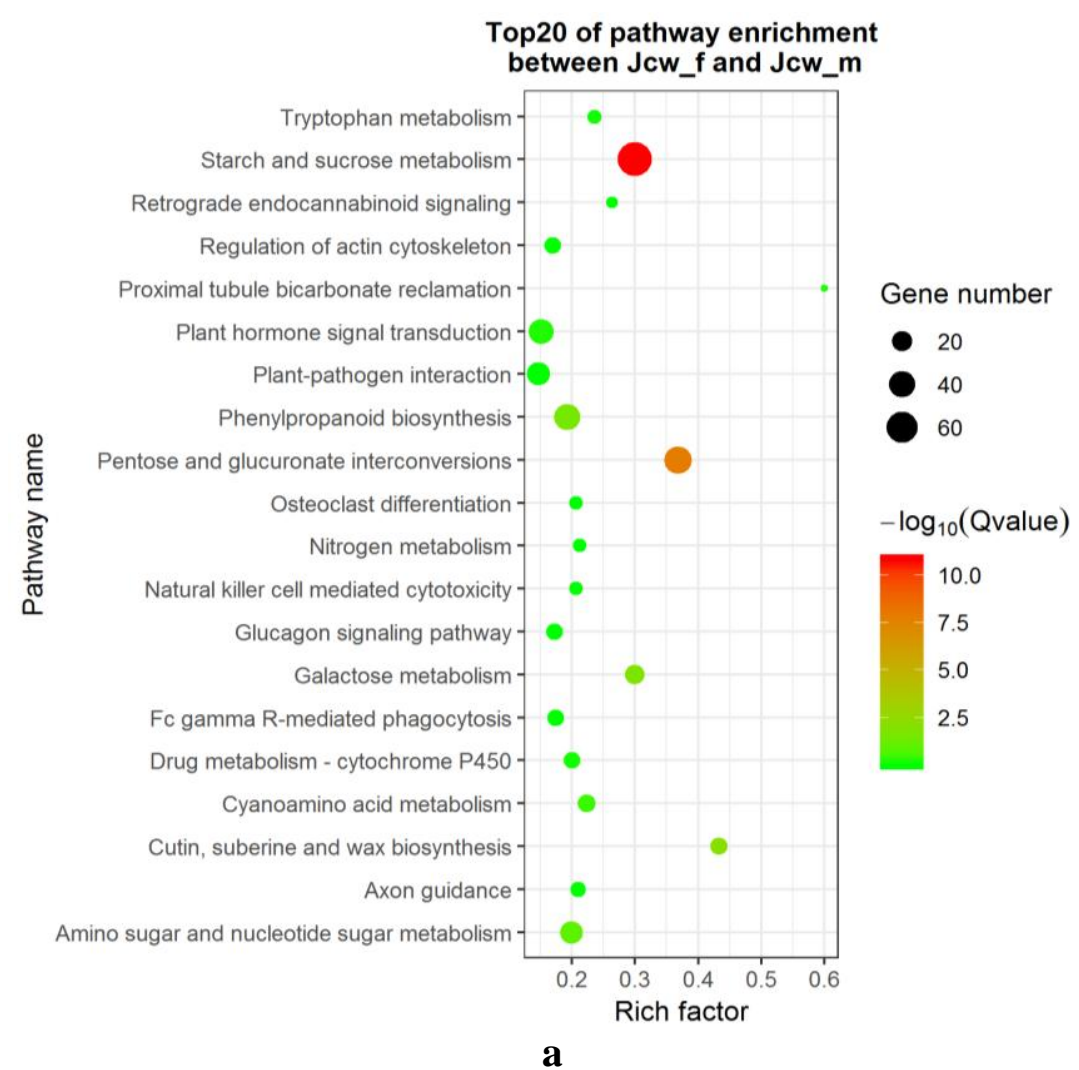




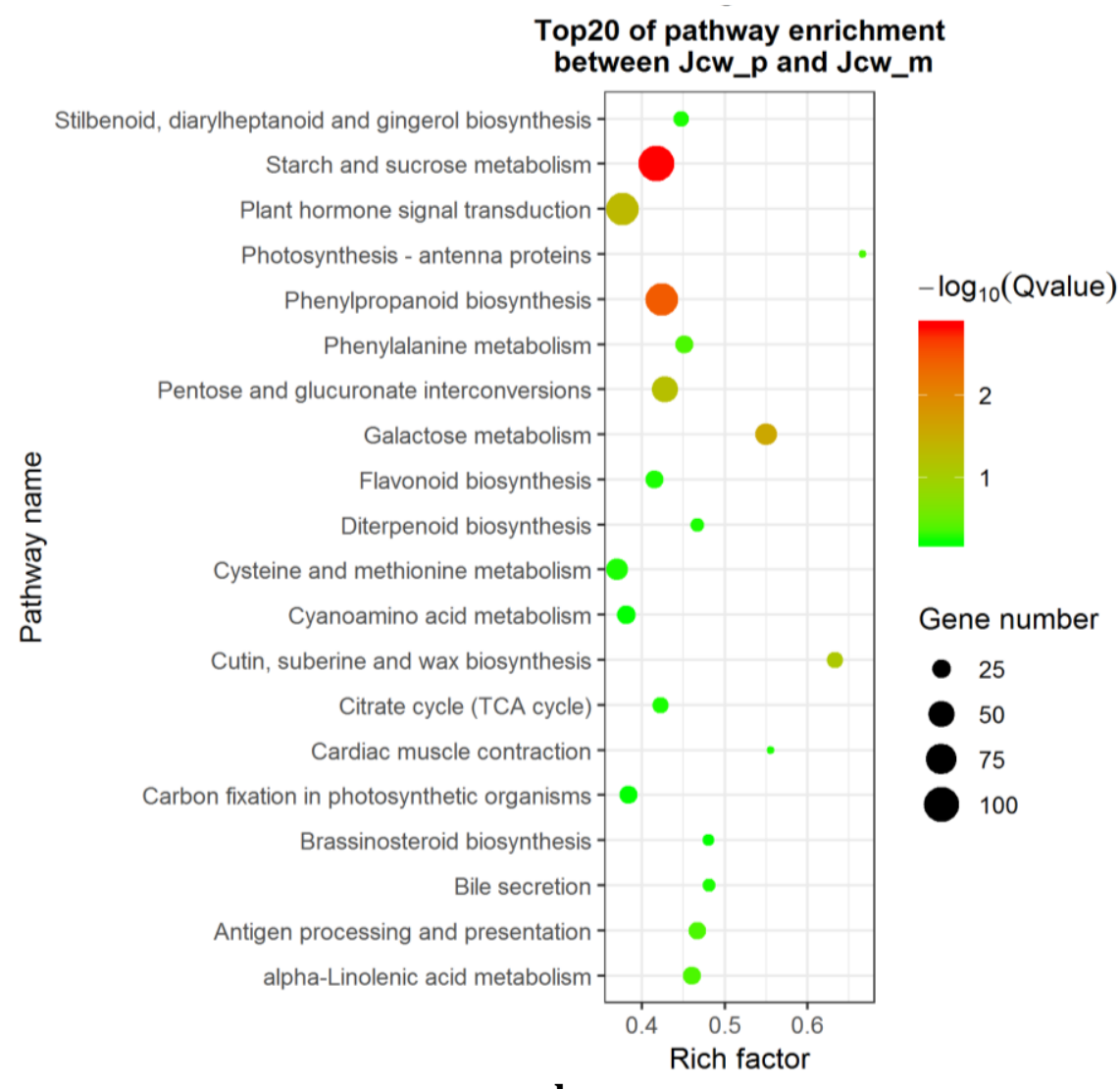

b

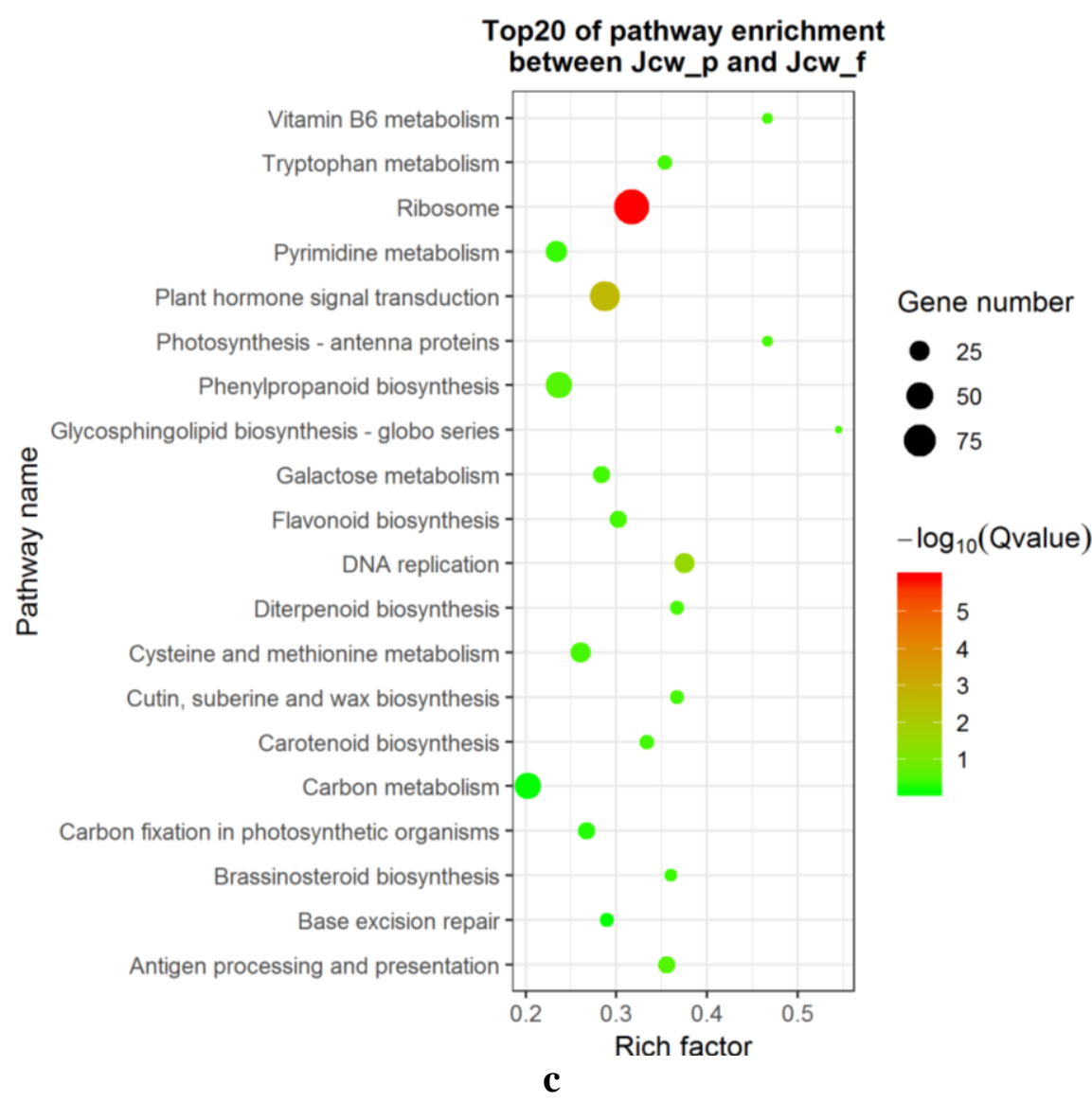

APPLIED ECOLOGY AND ENVIRONMENTAL RESEARCH 16(5):5595-5610. http://www.aloki.hu • ISSN 15891623 (Print) • ISSN 17850037 (Online)

DOI: http://dx.doi.org/10.15666/aeer/1605_55955610 (c) 2018, ALÖKI Kft., Budapest, Hungary 


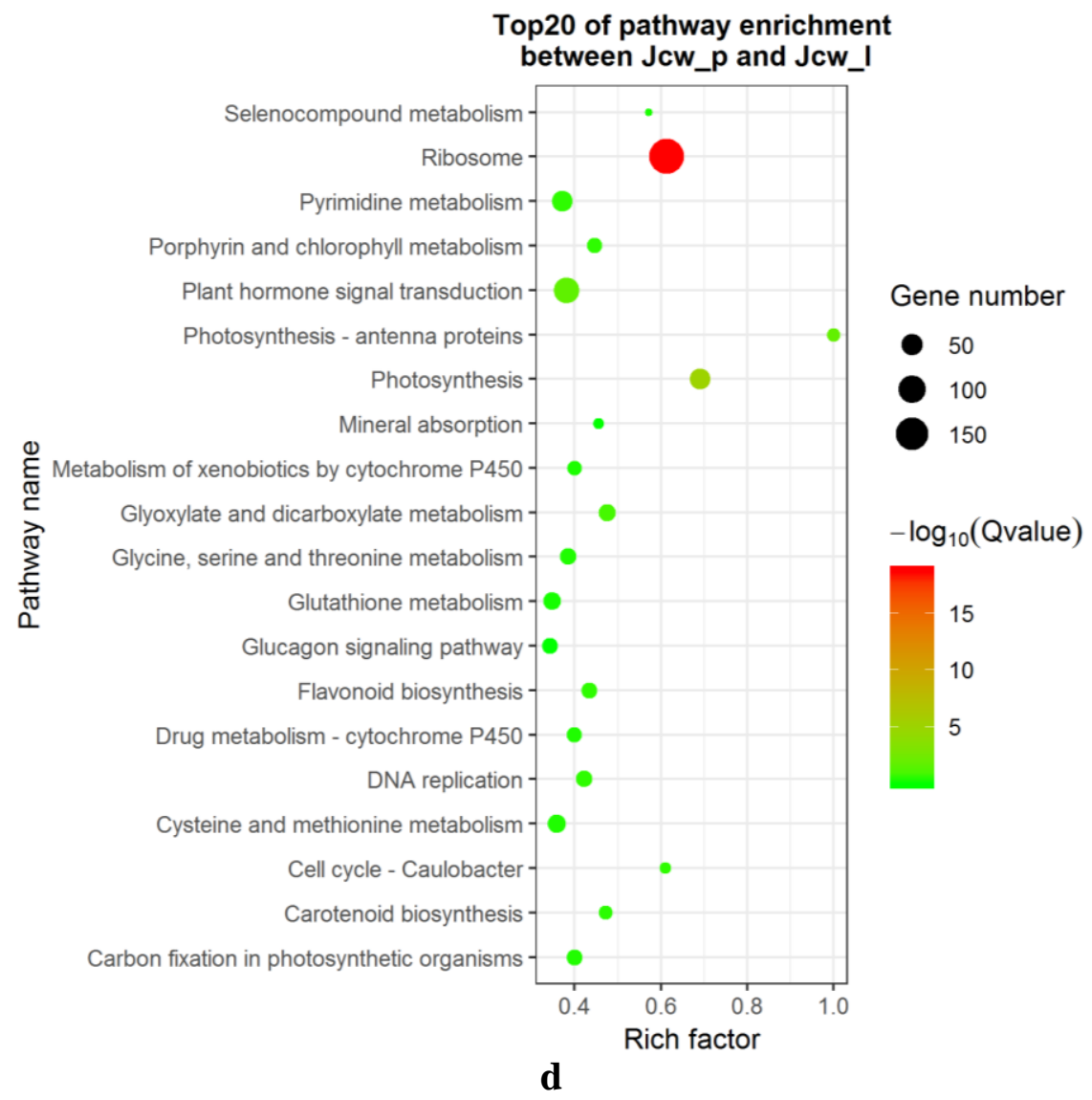

Figure 2. $\boldsymbol{a}$. Bubble map for the top 20 enriched pathways in female and male flowers. $\boldsymbol{b}$. Bubble map for the top 20 enriched pathways in inflorescence buds and male flowers. $c$. Bubble map for the top 20 enriched pathways in inflorescence buds and female flowers. d. Bubble map for the top 20 enriched pathways in inflorescence and leaf buds

\section{Changes in MADS-box gene expression}

Download the $J$. curcas proteinome data (http://www.ncbi.nlm.nih.gov/genome/915) (Sato et al., 2010) and the MADS-box Hidden Markov Model (HMM) model file (PF00319, http://pfam.sanger), using the HMM file as a seed, run HMM search in the $J$. curcas proteinome database (e-value < 1e-20). Construct a $J$. curcas-specific HMM model based on the screening results, then perform HMM search again (e-value $<0.01$ ), non-repetitive MADS-box related protein ID are extracted and the corresponding gene ID sequence and length information are extracted according to genomic annotation file. Search through the Pfam database, NCBI conserved domain database (https://www.ncbi.nlm.nih.gov/Structure/cdd/wrpsb.cgi) and SMART (http://smart.embl-heidelberg.de/) to confirm that all predicted genes contain the MADS-box domain. The MADS-box gene family is divided into two main lineages, type-I and type-II, that origi- nated from a duplication of ancestor genes during the divergence of plants and animals, the major difference between type-I and type-II genes is that type-II genes possess the K-box domain (Alvarez-Buylla et al., 2000; Theißen et al., 1996). Contained the K-box domain was identified as the type-II MADS-box gene, the otherwise is identified as the type-I MADS-box gene. Heatmap clustering and multiple annotations are performed using the circlize package ( $\mathrm{Gu}$ et al., 2014). Two 
types of MADS-box-related genes were subjected to hierarchical cluster analysis and visualized as four clusters according to the number of tissue samples (Fig. 3). The results show that for J. nigroviensrugosus, the MADS-box-related genes could be divided into two expression patterns (clustered by sample), one expressed in female and male flowers, and the other in inflorescence and leaf buds. In addition, the gene highly expressed in all samples is the type-II MADS-box gene.

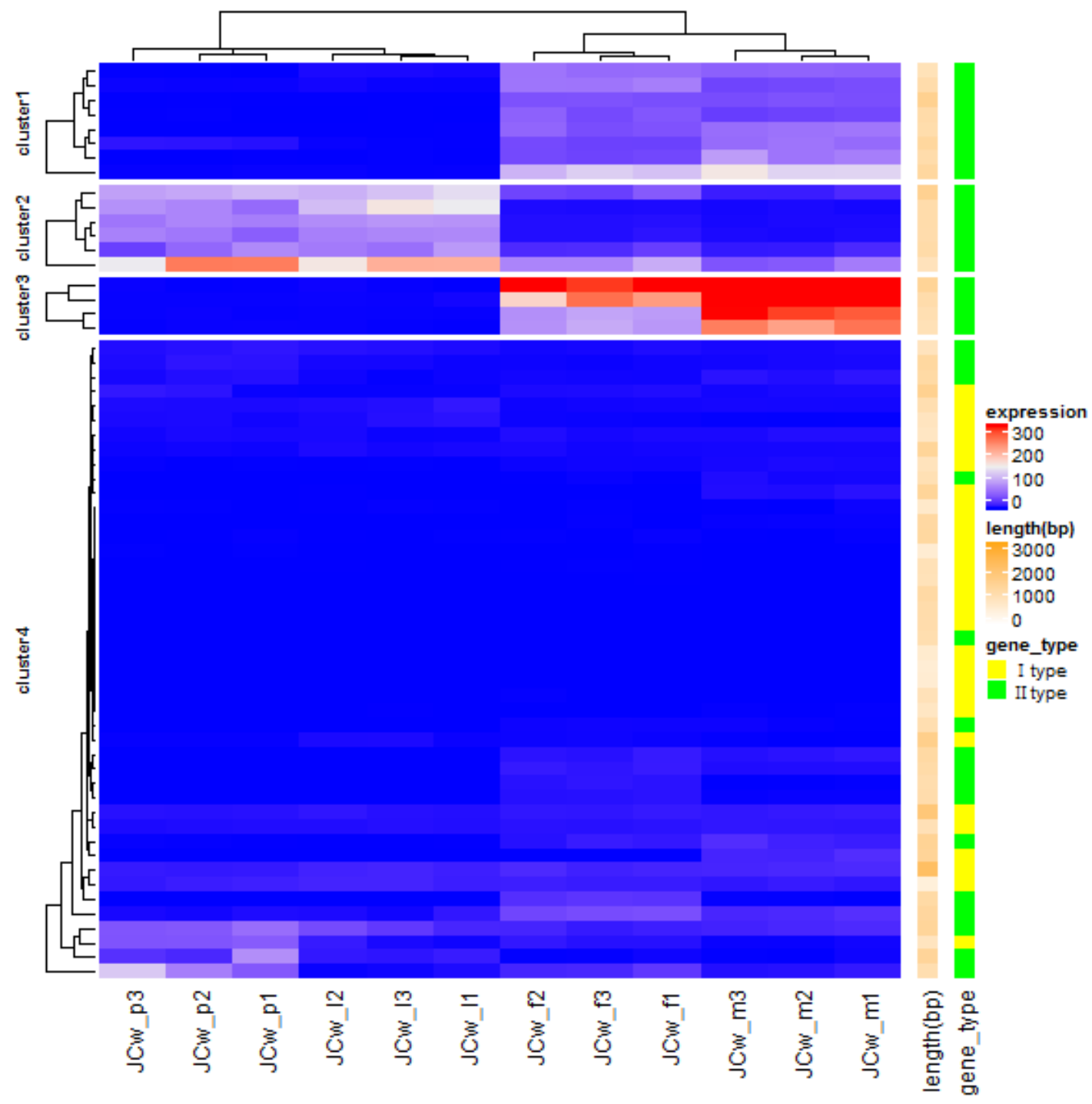

Figure 3. MADS-box gene expression profile of the four parts of J. nigroviensrugosus

\section{Discussion}

The low yield and low oil content of $J$. curcas seeds are the biggest problems encountered in the current planting of $J$. curcas trees; therefore, the identification of the most favorable genotypes is extremely important (Junqueira et al., 2016). As a new species, J. nigroviensrugosus has a higher female to male ratio and a higher seed yield than common $J$. curcas. Several papers have investigated flower induction of $J$. curcas in China and abroad, for instance, histological analysis of the differentiation of male and female flowers at different developmental stages (Xu et al., 2016), transcriptome 
differences between pure female and bisexual inflorescences (Chen et al., 2017), and gene responses subsequent to hormone induction (Ghosh et al., 2010; Pan et al., 2016). However, there is a lack of information regarding the flowering mechanism of $J$. nigroviensrugosus, and whether the relevant measures applied to the common J. curcas can be applied to J. nigroviensrugosus. Studies of $J$. nigroviensrugosus prior to and subsequent to inflorescence differentiation are beneficial for expanding knowledge of the sex determination mechanism of this energy-rich plant.

A plant undergoes a major physiological change during the transition from vegetative growth to reproductive development, and this transition is a result of responses to various endogenous and exogenous signals (Srikanth and Schmid, 2011). The expression of a specific gene, including the tissue specificity and the difference in transcriptional level, may influence the sex determination of a flower (Ramos et al., 2017). The differentially-expressed genes in the comparison group of female and male flowers may be involved in the regulation of gender differentiation. During this period, carbohydrates may act as mobile signals to regulate flower morphogenesis (Turnbull, 2011; Corbesier et al., 2006). Sugar catabolism enzymes may increase the assimilation ability of the shoots, thereby accelerating shoot growth and increasing primordium numbers (Ito et al., 2002). Low concentrations of sucrose may promote flowering, whereas high concentrations may delay flowering (Ohto et al., 2001). Sucrose-mediated signaling is integrated into the photoperiod pathway, located downstream of the CONSTANS (CO) gene and upstream of the FLOWERING LOCUS T (FT) gene (Seo et al., 2011). Sucrose-phosphate synthase (SPS) (EC: 2.4.1.14) is considered to be a key enzyme in the control of sucrose synthesis (Sawitri et al., 2018). With respect to inflorescence buds and male flowers, the SPS-related genes (gene 7836, gene 12091 and gene 14471) were upregulated in male flowers. In addition, polygalacturonase (PG) was involved in the development of pollen (Ogawa et al., 2009), whichwas significantly upregulated in male flowers. The distribution of sugars and starch is known to be related to flower transformation. Studies conducted on various angiosperm species have shown a correlation between flower abortion and starch content (Reale et al., 2009). Starch is composed of linear and branched glucose (Glc) polymers (Zeeman et al., 2010). The soluble starch synthase (EC: 2.4.1.21) is primarily related to amylopectin catalysis, while granule-bound starch synthase (EC: 2.4.1.242) is primarily associated with the production of amylose, both of which were found to be highly expressed in the male flowers of J. nigroviensrugosus. This data indicates that high expression of sucrose and starch synthesis-related genes plays an important role in the development of male flowers in J. nigroviensrugosus.

Plant hormones play essential roles in gender determination (Aryal and Ming, 2014). Auxin plays a dominant role in floral organ initiation and organogenesis (Chandler, 2011), and CTK triggers the initiation of female flower primordium (Hui et al., 2017). Application of exogenous cytokinin (6-benzyladenine, BA) on inflorescence buds can significantly increase the number of female flowers, BA treatment may delay floral organ formation by inhibiting the transcription of the A, B and E classes of floral organidentity genes, which allowing extra time for the plant to produce more primordia in inflorescence meristems (Chen et al., 2014). Type-B Arabidopsis response regulators (ARRs) are positive regulators of the CTX response (Mason et al., 2005), and type-A ARRs are thought to work as negative regulators of CTX to inhibit the activity of typeB ARRs (To and Kieber, 2008; To et al., 2004). B-ARR (gene 8030 and gene 17670) was significantly upregulated in inflorescence buds compared with leaf buds. 
Comparing the male and female flowers, A-ARR (gene 13354) was significantly upregulated in female flowers, whereas B-ARR (gene 20147) was significantly upregulated in male flowers. These data suggest that the initial development of $J$. nigroviensrugosus female flower primordia requires the induction of CTK, but the late developmental stage only needs a low concentration of CTK. As a gaseous plant hormone, ethylene regulates the flowering time of plants through interactions with other plant hormones (Iqbal et al., 2017). In J. curcas, treatment with a low concentration of ethephon $(25 \mathrm{ppm})$ is thought to increase the number of female flowers (Makwana and Robin, 2013), and increasing the ethephon concentration has been shown to decrease the number of male and female flowers per inflorescence as well as the total flower number (Costa et al., 2016). Compared with inflorescence buds and male flowers, the expression of ERF1/2 (gene 18694) was significantly increased in female flowers of $J$. nigroviensrugosus, highlighting the importance of ethylene in the regulation of female flower formation and development. In the ABA signaling pathway, the SnRK2 signal plays a crucial role in the sex determination of ferns and regulates the sex ratio of male to hermaphrodite in the reproductive cycle (McAdam et al., 2016). However, there is no related report regarding the impact of SnRK2 on the female to male flower ratio of angiosperms, especially $J$. curcas.

The differential expression of transcription factors (TFs), also known as trans-acting factors, play an essential role in the control of organ development (Latchman, 1997). Six signaling pathways have been identified to regulate the flowering of A. thaliana: photoperiod, vernalization, autonomous, gibberellin (GA), temperature-sensitive, and age-dependent control (Fornara et al., 2010). These signaling pathways are both independent and connected, and converge the flowering signals to several key integrators such as FT, TWIN SISTER OF FT (TSF) and SUPPRESSOR OF OVEREXPRESSION OF CONSTANS 1 (SOC1), activating the expression of meristem-specific genes such as LEAFY (LFY), APETALA1 (AP1) and FRUITFULL (FUL), eventually initiating plant flowering (Cho et al., 2017). Among these, the expression of SOC1 was high in leaf and inflorescence buds, but low in male and female flowers. Conversely, FT was highly expressed in male and female floral organs. The zinc finger transcription factor CONSTANS contains a B-box domain (Gangappa et al., 2014), which plays a central role in photoperiod sensing (Lee et al., 2017). The expression of CONSTANS-LIKE 7 (gene 24482) and CONSTANS-LIKE 3 (gene 12833) were upregulated in both male and female flowers, with respect to inflorescence buds.

NAC genes encode plant-specific transcription factors that are closely related to vegetative reproduction, and embryo and flower development (Olsen et al., 2005). CUPSHAPED COTYLEDON 1 (CUC1), CUC2 and CUC3 have been shown to regulate organ segregation and meristem formation (Vroemen et al., 2003). In addition to controlling shoot meristem activity, $\mathrm{CUC1}$ and CUC2 also play key roles in female organ development (Kamiuchi et al., 2014). Genetic interaction analyses using single, double and triple mutants of cuc1-1D, cuc2-1D (a CUC2 mutant similar to cuc1-1D), and hws-1, demonstrate that HWS (HAWAIIAN SKIRT), CUC1 and CUC2 act together to control floral organ number (González-Carranza et al., 2017). It is believed that CUC2 is associated with female flower opening of $J$. curcas (Gangwar et al., 2016). Through comparison of female and male flowers of $J$. nigroviensrugosus, we found nine NAC-related genes upregulated in female flowers (including homologous genes of CUC2 and CUC3), and 12 NAC-related genes upregulated in male flowers (including 
NAC2 and NAC29). These data indicate that CUC2 and CUC3 make a contribution in the formation and development of J. nigroviensrugosus female flowers.

The MADS-box TFs are the primary components of the current well-known ABCDE model which are closely related to the origin and evolution of plant reproductive organs (Smaczniak et al., 2012; Theissen et al., 2000). The MADS-box TFs play a pivotal role in floristic differentiation (Theissen and Melzer, 2007). Ming et al. (2011) showed that the structural determinants of floral organs are randomly distributed in autosomes, therefore only the spatiotemporal patterns are expressed between sexes, but the expression are not sex-specific. With respect to the DEGs selected in the comparison group of female and male flowers in J. nigroviensrugosus, seven genes including ANR1, AGL14 and AGL7 were upregulated in female flowers, while 11 genes including AGL11, AGL62 and AGL20/SOC1 were upregulated in male flowers. The Band C-class genes, the characteristic genes of flower organ development (Pařenicová et al., 2003), and the dependent SEP1, SEP2 and SEP3 genes, were all similarly expressed in both female and male floral organs of J. nigroviensrugosus, and the expression was not sex-specific. The MADS-box-related genes from the four different tissues of $J$. nigroviensrugosus were primarily divided into two expression patterns (Fig. 3). Cluster 3-related genes were expressed only in male and female flowers, while cluster 2-related genes were expressed in inflorescences and leaf buds, with a certain degree of conservativity. Two MADS-box-related genes in cluster 3 were only highly expressed in male flowers, spatiotemporally. In addition, the gene highly expressed in all samples belongs to the type-II MADS-box gene. Several cluster 4-related genes, including AGL62 and AGL80, were not expressed in any of the four tissues, suggestive of involvement in early endosperm development (Kang et al., 2008; Portereiko et al., 2006) and regulation of certain physiological activities.

\section{Conclusion}

As a new cultivar, transcriptome sequencing of inflorescence and leaf bud differentiation in J. nigroviensrugosus was beneficial to analyze the nutrient supply relationship and hormonal regulation of different plant parts. Genes related to sucrose phosphate synthase, soluble starch synthase and granule-bound starch synthase were highly expressed in male flowers of $J$. nigroviensrugosus, suggestive of an important role in the formation and development of male flowers. Initial development of female flower primordia required the induction of CTK, while late stage development only required a low concentration of CTK in coordination with other hormones. CUC2 and CUC3 were very important in the formation and development of female flowers of $J$. nigroviensrugosus. As the most important transcription factors, MADS-box-related genes were conserved throughout different parts of J. nigroviensrugosus. Moreover, a number of DEGs were screened during the differentiation of male and female flowers, this study provided an important basis for further research regarding the differentiation of flowers and the molecular mechanism of high-yield J. nigroviensrugosus breeding.

Acknowledgements. The work was supported by National Natural Science Foundation of China-Study on flower bud differentiation and flowering mechanism of Jatropha nigroviensrugosus CV Yang. (31360165) 


\section{REFERENCES}

[1] Alvarez-Buylla, E. R., Pelaz, S., Liljegren, S. J., Gold, S. E., Burgeff, C., Ditta, G. S., de Pouplana, L. R., Martínez-Castilla, L., Yanofsky, M. F. (2000): An ancestral MADS-box gene duplication occurred before the divergence of plants and animals. - Proceedings of the National Academy of Sciences 97(10): 5328-5333.

[2] Aryal, R., Ming, R. (2014): Sex determination in flowering plants: papaya as a model system. - Plant Science 217: 56-62.

[3] Ashoke, B., Kalyani, D., Datta, S. K. (2005): Floral biology, floral resource constraints and pollination limitation in Jatropha curcas L. - Pakistan Journal of Biological Sciences 8(3): 456-460.

[4] Chandler, J. W. (2011): The hormonal regulation of flower development. - Journal of Plant Growth Regulation 30(2): 242-254.

[5] Chen, M. S., Pan, B. Z., Wang, G. J., Ni, J., Niu, L., Xu, Z. F. (2014): Analysis of the transcriptional responses in inflorescence buds of Jatropha curcas exposed to cytokinin treatment. - BMC Plant Biology 14(1): 318.

[6] Chen, M. S., Pan, B. Z., Fu, Q., Tao, Y. B., Martínez-Herrera, J., Niu, L., Ni, J., Dong, Y. L., Zhao, M. L., Xu, Z. F. (2017): Comparative transcriptome analysis between gynoecious and monoecious plants identifies regulatory networks controlling sex determination in Jatropha curcas. - Frontiers in Plant Science 7: 1953.

[7] Cho, L. H., Yoon, J., An, G. (2017): The control of flowering time by environmental factors. - The Plant Journal 90(4): 708-719.

[8] Corbesier, L., Coupland, G. (2006): Coupland. The quest for florigen: a review of recentprogress. - Journal of Experimental Botany 57(13): 3395-3403.

[9] Costa, A. P., Vendrame, W., Nietsche, S., Crane, J., Moore, K., Schaffer, B. (2016): Branching, flowering and fruiting of Jatropha curcas treated with ethephon or benzyladenine and gibberellins. - Anais da Academia Brasileira de Ciências 88(2): 989998.

[10] De Lourdes Adriano-Anaya, M., Pérez-Castillo, E., Salvador-Figueroa, M., RuizGonzález, S., Vázquez-Ovando, A., Grajales-Conesa, J., Ovando-Medina, I. (2016): Sex expression and floral diversity in Jatropha curcas: a population study in its center of origin. - PeerJ 4: e2071.

[11] Dias, L. A. S., Missio, R. F., Dias, D. C. F. S. (2012): Antiquity, botany, origin and domestication of Jatropha curcas (Euphorbiaceae), a plant species with potential for biodiesel production. - Genet. Mol. Res 11(3): 2719-28.

[12] Fornara, F., de Montaigu, A., Coupland, G. (2010): SnapShot: control of flowering in Arabidopsis. - Cell 141(3): 550-550.

[13] Gangappa, S. N., Botto, J. F. (2014): The BBX family of plant transcription factors. Trends in Plant Science 19(7): 460-470.

[14] Gangwar, M., Sood, H., Chauhan, R. S. (2016): Genomics and relative expression analysis identifies key genes associated with high female to male flower ratio in Jatropha curcas L. - Molecular Biology Reports 43(4): 305-322.

[15] Gangwar, M., Sood, A., Bansal, A., Chauhan, R. S. (2018): Comparative transcriptomics reveals a reduction in carbon capture and flux between source and sink in cytokinintreated inflorescences of Jatropha curcas L. - Biotech 8(1): 64.

[16] Gao, W. J., Li, S. F., Zhang, G. J., Wang, N. N., Deng, C. L., Lu, L. D. (2013): Comparative analysis of gene expression by microarray analysis of male and female flowers of Asparagus officinalis. - Bioscience, Biotechnology, and Biochemistry 77(6): 1193-1199.

[17] Ghosh, A., Chikara, J., Chaudhary, D. R., Prakash, A. R., Boricha, G., Zala, A. (2010): Paclobutrazol arrests vegetative growth and unveils unexpressed yield potential of Jatropha curcas. - Journal of Plant Growth Regulation 29(3): 307-315. 
[18] González-Carranza, Z. H., Zhang, X., Peters, J. L., Boltz, V., Szecsi, J., Bendahmane, M., Roberts, J. A. (2017): HAWAIIAN SKIRT controls size and floral organ number by modulating CUC1 and CUC2 expression. - PloS One 12(9): e0185106.

[19] Gu, Z., Gu, L., Eils, R., Schlesner, M., Brors, B. (2014): Circlize implements and enhances circular visualization in R. - Bioinformatics 30(19): 2811-2812.

[20] He, H., Wang, X. R., Hu, Z. (2016): Nutritional transport and photosynthetic characteristics of Guizhou Jatropha nigroviensrugosus and ordinary Jatropha curcas. Journal of West China Forestry Science 45(5): 79-83.

[21] Hui, W., Yang, Y., Wu, G., Peng, C., Chen, X., Zayed, M. Z. (2017): Transcriptomeprofile analysis reveals the regulation mechanism of floral sex differentiation in Jatropha curcas L. - Scientific Reports 7(1): 16421.

[22] Iqbal, N., Khan, N. A., Ferrante, A., Trivellini, A., Francini, A., Khan, M. I. R. (2017): Ethylene role in plant growth, development and senescence: interaction with other phytohormones. - Frontiers in Plant Science 8: 475.

[23] Ito, A., Hayama, H., Kashimura, Y. (2002): Sugar metabolism in buds during flower bud formation: a comparison of two Japanese pear [Pyrus pyrifolia (Burm.) Nak.] cultivars possessing different flowering habits. - Scientia Horticulturae 96(1-4): 163-175.

[24] Junqueira, V. S., de Azevedo Peixoto, L., Laviola, B. G., Bhering, L. L., Mendonça, S., Costa, T. D. S. A., Antoniassi, R. (2016): Bayesian Multi-Trait Analysis Reveals a Useful Tool to Increase Oil Concentration and to Decrease Toxicity in Jatropha curcas L. - PloS One 11(6): e0157038.

[25] Kamiuchi, Y., Yamamoto, K., Furutani, M., Tasaka, M., Aida, M. (2014): The CUC1 and CUC2 genes promote carpel margin meristem formation during Arabidopsis gynoecium development. - Frontiers in Plant Science 5: 165.

[26] Kang, I. H., Steffen, J. G., Portereiko, M. F., Lloyd, A., Drews, G. N. (2008): The AGL62 MADS domain protein regulates cellularization during endosperm development in Arabidopsis. - The Plant Cell 20(3): 635-647.

[27] Kaushik, N., Kumar, K., Kumar, S., Kaushik, N., Roy, S. (2007): Genetic variability and divergence studies in seed traits and oil content of Jatropha (Jatropha curcas L.) accessions. - Biomass and Bioenergy 31(7): 497-502.

[28] Latchman, D. S. (1997): Transcription factors: an overview. - The International Journal of Biochemistry \& Cell Biology 29(12): 1305-1312.

[29] Lee, B. D., Kim, M. R., Kang, M. Y., Cha, J. Y., Han, S. H., Nawkar, G. M., Kim, W. Y. (2017): The F-box protein FKF1 inhibits dimerization of COP1 in the control of photoperiodic flowering. - Nature Communications 8(1): 2259.

[30] Makwana, V., Robin. P. (2013): Interaction between GA and ethrel in inducing female flowers in Jatropha curcas. - Int. J. Biotechnol. Bioeng. Res. 4: 465-472.

[31] Mao, Y., Liu, W., Chen, X., Xu, Y., Lu, W., Hou, J., Wu, L. (2017): Flower development and sex determination between male and female flowers in Vernicia fordii. - Frontiers in Plant Science 8: 1291.

[32] Mason, M. G., Mathews, D. E., Argyros, D. A., Maxwell, B. B., Kieber, J. J., Alonso, J. M., Schaller, G. E. (2005): Multiple type-B response regulators mediate cytokinin signal transduction in Arabidopsis. - The Plant Cell 17(11): 3007-3018.

[33] McAdam, S. A., Brodribb, T. J., Banks, J. A., Hedrich, R., Atallah, N. M., Cai, C., Geiger, D. (2016): Abscisic acid-controlled sex before transpiration in vascularplants. Proceedings of the National Academy of Sciences 113(45): 12862-12867.

[34] Ming, R., Bendahmane, A., Renner, S. S. (2011): Sex chromosomes in land plants. Annual Review of Plant Biology 62: 485-514.

[35] Montes, J. M., Melchinger, A. E. (2016): Domestication and breeding of Jatropha curcas L. - Trends Plant Sci 21(12): 1045-1057.

[36] Ogawa, M., Kay, P., Wilson, S., Swain, S. M. (2009): ARABIDOPSISDEHISCENCE ZONE POLYGALACTURONASE1 (ADPG1), ADPG2, and QUARTET2 are 
polygalacturonases required for cell separation during reproductive development in Arabidopsis. - The Plant Cell 21(1): 216-233.

[37] Ohto, M. A., Onai, K., Furukawa, Y., Aoki, E., Araki, T., Nakamura, K. (2001): Effects of sugar on vegetative development and floral transition in Arabidopsis. - Plant Physiology 127(1): 252-261.

[38] Olsen, A. N., Ernst, H. A., Leggio, L. L., Skriver, K. (2005): NAC transcription factors: structurally distinct, functionally diverse. - Trends in Plant Science 10(2): 79-87.

[39] Pan, B. Z., Luo, Y., Song, L., Chen, M. S., Li, J. L., Xu. Z. F. (2016): Thidiazuron increases fruit number in the biofuel plant Jatropha curcas by promoting pistil development. - Industrial Crops and Products 81: 202-210.

[40] Pařenicová, L., de Folter, S., Kieffer, M., Horner, D. S., Favalli, C., Busscher, J., Angenent, G. C. (2003): Molecular and phylogenetic analyses of the complete MADSbox transcription factor family in Arabidopsis: new openings to the MADS world. - The Plant Cell 15(7): 1538-1551.

[41] Portereiko, M. F., Lloyd, A., Steffen, J. G., Punwani, J. A., Otsuga, D., Drews, G. N. (2006): AGL80 is required for central cell and endosperm development in Arabidopsis. The Plant Cell 18(8): 1862-1872.

[42] Ramos, M. J. N., Coito, J. L., Fino, J., Cunha, J., Silva, H., de Almeida, P. G., CostaSara, M. M. R., Amâncio, S., Paulo, O. S., Rocheta, M. (2017): Deep analysis of wild Vitis flower transcriptome reveals unexplored genome regions associated with sex specification. - Plant Molecular Biology 93(1-2): 151-170.

[43] Reale, L., Sgromo, C., Ederli, L., Pasqualini, S., Orlandi, F., Fornaciari, M., Ferranti, F., Romano, B. (2009): Morphological and cytological development and starch accumulation in hermaphrodite and staminate flowers of olive (Olea europaea L.). - Sexual Plant Reproduction 22(3): 109-119.

[44] Rocheta, M., Sobral, R., Magalhães, J., Amorim, M. I., Ribeiro, T., Pinheiro, M., Egas, C., Morais-Cecílio, L., Costa, M. M. (2014): Comparative transcriptomic analysis of male and female flowers of monoecious Quercus suber. - Frontiers in Plant Science 5: 599.

[45] Sato, S., Hirakawa, H., Isobe, S., Fukai, E., Watanabe, A., Kato, M., Kawashima, K., Minami, C., Muraki, A., Nakazaki, N., Takahashi, C. (2010): Sequence analysis of the genome of an oil-bearing tree, Jatropha curcas L. - DNA Research 18(1): 65-76.

[46] Sawitri, W. D., Afidah, S. N., Nakagawa, A., Hase, T., Sugiharto, B. (2018): Identification of UDP-glucose binding site in glycosyltransferase domain of sucrose phosphate synthase from sugarcane (Saccharum officinarum) by structure-based sitedirected mutagenesis. - Biophysical Reviews 10(2): 1-6.

[47] Seo, P. J., Ryu, J., Kang, S. K., Park, C. M. (2011): Modulation of sugar metabolism by an INDETERMINATE DOMAIN transcription factor contributes to photoperiodic flowering in Arabidopsis. - The Plant Journal 65(3): 418-429.

[48] Smaczniak, C., Immink, R. G., Angenent, G. C., Kaufmann, K. (2012): Developmental and evolutionary diversity of plant MADS-domain factors: insights from recent studies. Development 139(17): 3081-3098.

[49] Srikanth, A., Schmid, M. (2011): Regulation of flowering time: all roads lead to Rome. Cellular and Molecular Life Sciences 68(12): 2013-2037.

[50] Theissen, G., Melzer, R. (2007): Molecular mechanisms underlying origin and diversification of the angiosperm flower. - Annals of Botany 100(3): 603-619.

[51] Theißen, G., Kim, J. T., Saedler, H. (1996): Classification and phylogeny of the MADSbox multigene family suggest defined roles of MADS-box gene subfamilies in the morphological evolution of eukaryotes. - Journal of Molecular Evolution 43(5): 484-516.

[52] Theissen, G., Becker, A., Di Rosa, A., Kanno, A., Kim, J. T., Münster, T., Winter, K., Saedler, H. (2000): A Short History of MADS-Box Genes in Plants. - In: Doyle, J. J., Gaut, B. S. (eds.) Plant Molecular Evolution, pp. 115-149. Springer, Dordrecht.

[53] To, J. P., Kieber, J. J. (2008): Cytokinin signaling: two-components and more. - Trends in Plant Science 13(2): 85-92. 
[54] To, J. P., Haberer, G., Ferreira, F. J., Deruère, J., Mason, M. G., Schaller, G. E., Alonso, J. M., Ecker, J. R., Kieber, J. J. (2004): Type-A Arabidopsis response regulators are partially redundant negative regulators of cytokinin signaling. - The Plant Cell 16(3): 658-671.

[55] Turnbull, C. (2011): Long-distance regulation of flowering time. - Journal of Experimental Botany 62(13): 4399-4413.

[56] Vroemen, C. W., Mordhorst, A. P., Albrecht, C., Kwaaitaal, M. A., de Vries, S. C. (2003): The CUP-SHAPED COTYLEDON3 gene is required for boundary and shoot meristem formation in Arabidopsis. - The Plant Cell 15(7): 1563-1577.

[57] Xu, G., Huang, J., Yang, Y., Yao, Y. A. (2016): Transcriptome analysis offlower sex differentiation in Jatropha curcas L. using RNA sequencing. - PloS One 11(2): $\mathrm{e} 0145613$.

[58] Yang, C. (2013): Phenotypic Characteristics and Genetic Stability of New Varieties of Jatropha curcas. - Botany in Ecological Civilization: Present and Future - The Fifteenth Congress of Chinese Society of Botany and the 80th Academic Year Symposium. Session 4 Session: Resource Botany. http://cpfd.cnki.com.cn/Article/CPFDTOTALZGZO201310005036.htm.

[59] Yang, C. Y., Fang, Z., Li, B., Long, Y. F. (2012): Review and prospects of Jatropha biodiesel industry in China. - Renewable and Sustainable Energy Reviews 16(4): 21782190.

[60] Yang, C. Y., Lu, W., Wu, X., Xu, Z., Long, Y. (2015): Analysis on components of fatty acids from new Jatropha cultivars seed oils and their potential of preparing biodiesel. Botanical Research 4(1): 16-24.

[61] Zeeman, S. C., Kossmann, J., Smith, A. M. (2010): Starch: its metabolism, evolution, and biotechnological modification in plants. - Annual Review of Plant Biology 61: 209-234. 